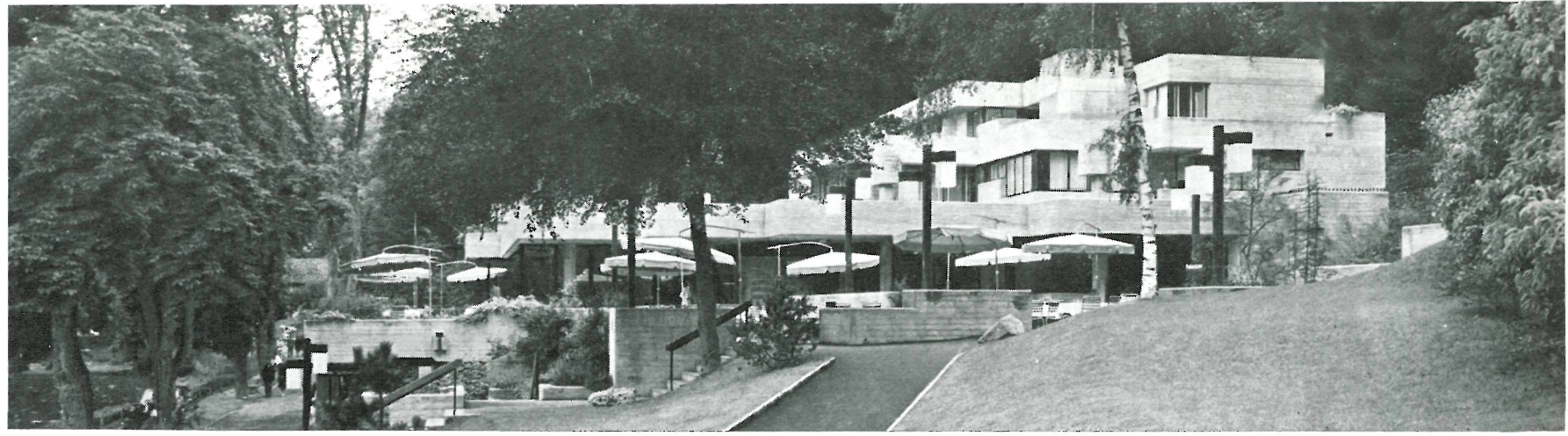

\title{
restaurante PARK en Neuhausen - Suiza
}

FOERDERER \& ZWIMPFER, arquitectos

(Estudio: FOERDERER \& OTTO \& ZWIMPFER)

\section{sinopsis}

Se observa en este restaurante el sello característico del arquitecto-escultor Walter Foerderer, con sus combinaciones hormigon-madera y sus formas senta una estudiada fison edício prenada, consecuencia de la perfecta adaptación al terreno, lo que constituye, además, uno de sus más singulares atractivos.

Funciona todo el año. Sus locales cubiertos tienen capacidad para servir 50 comidas; y al aire libre, en las terrazas -que se utilizan durante la atendidos, en cada turno, unos 200 co. mensales. Constituye un lugar de parada agradable para los visitantes, en sus excursiones, ofreciéndoles puntos de vista apropiados desde los que pueden contemplar el excepcional panora-
Próximo a una de las bellísimas cascadas existentes en el Rhin, y no lejos de la orilla más oriental del lago Constanza, ha sido construido este restaurante, que, además de venir a satisfacer una necesidad turística, sirve a los habitantes de Neuhausen, sobre todo en los meses no pertenecientes a la temporada turística, ya que dispone de comedores, con un total de 50 plazas en locales cerrados; además de los existentes, para 200 plazas, al aire libre, en las terrazas, utilizados en los meses de verano.

Desde hace algunos años la firma de arquitectos autora del proyecto se ha distinguido en el campo del diseño y realización de edificios escolares con un aspecto característico; son las mismas «formas macizas y consistentes» empleadas en aquéllos, las que aparecen también evidentes en este edificio destinado a restaurante.

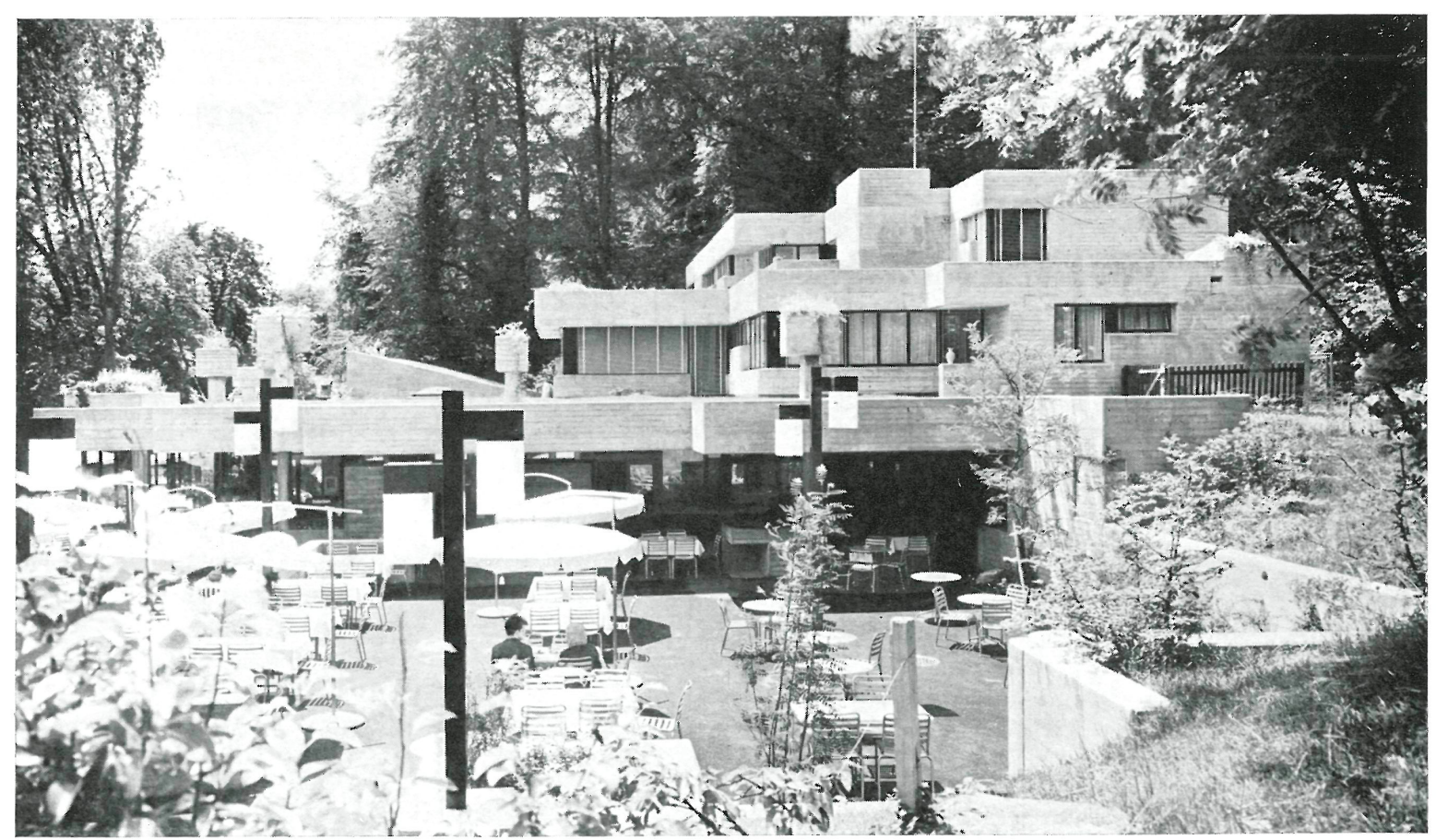


Pero mientras las escuelas inspiraban una cierta sensación de respeto y seriedad; el restaurante está impregnado de una atmósfera animada, reconociéndose en él inmediatamente la mano del arquitecto-escultor Walter Foerderer, no sólo en la composición de los elementos del edificio, sino también en la yuxtaposición de sus dos materiales favoritos: madera y hormigón.

Al proyectar este restaurante, los autores se abstuvieron de emplear ornamentaciones llamativas; todos sus esfuerzos se encaminaron a proporcionar a los visitantes en las excursiones de verano un lugar de parada agradable y tranquilo, a ofrecerles una serie de terrazas escalonadas desde las que disfrutasen contemplando las espectaculares cascadas, y a crear ambientes interiores adecuadamente acondicionados para que la población de la localidad pudiera utilizarlos durante la temporada de invierno, permitiendo así la explotación del restaurante a lo largo de todo el año.

Durante la mayor parte del verano, las comidas son servidas -al aire libre - en las terrazas, que ascienden por la ladera adaptándose al perfil del terreno, y crean una transición, afortunada, naturaleza-arquitectura.
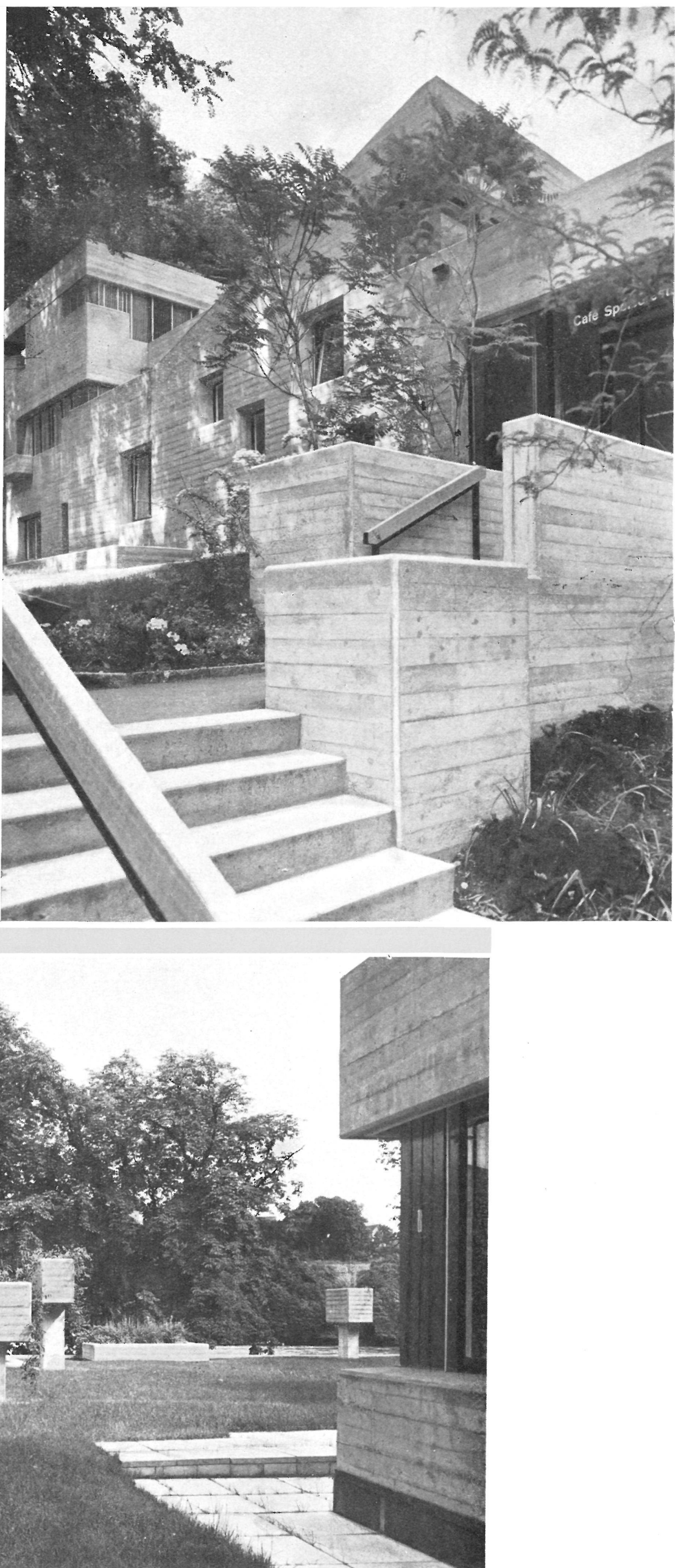
plantas

\section{bojo} príncína

13. Restaurante.

14. Servicio.

15. Cocina.

16. Oficina.

8. Refrigeradores.

9. Almacén de bebidas.

20. Almacén de sillas y mesas.

21. Asientos en terraza.

\section{bajo inferior}

1. Kiosco existente.-2. Almacén del kiosco.-3. W. C. restaurante.-4. W. C. público.-5. Lavadero.-6. Calefaccion.-7. Depósitos.-8. Guardarropas (empleados).-9. Ascensor.-10. Almacén restaurante.-11. Refugios.-12. Garaje.

\section{sección}
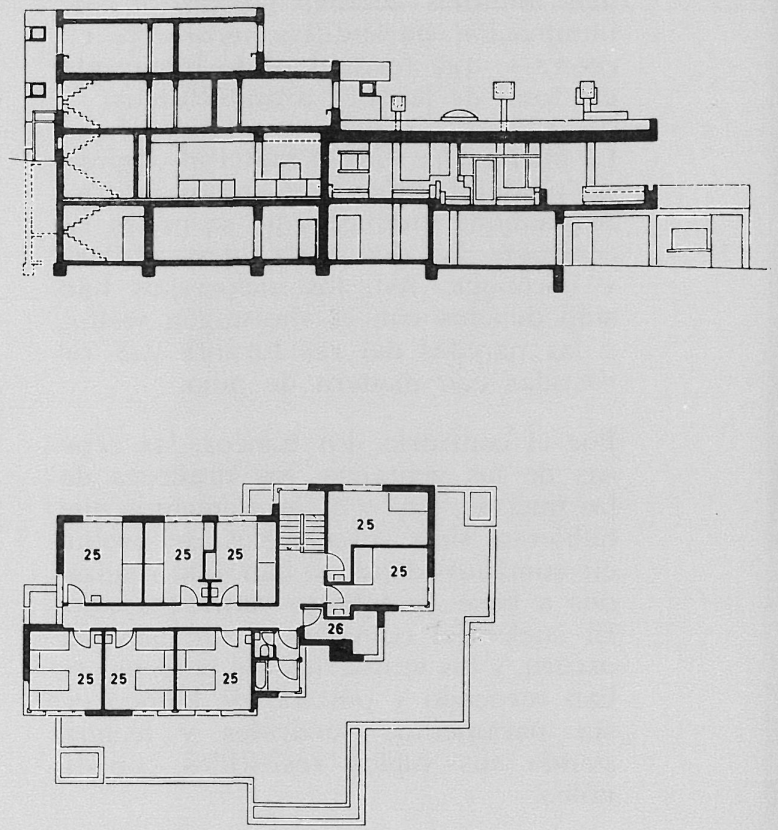

\section{Segumda}

25. Cuartos (empleados).

26. Cuartos de máquinas.

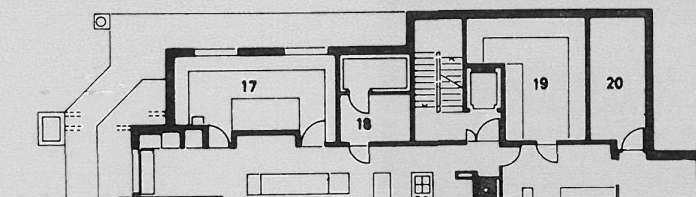

2
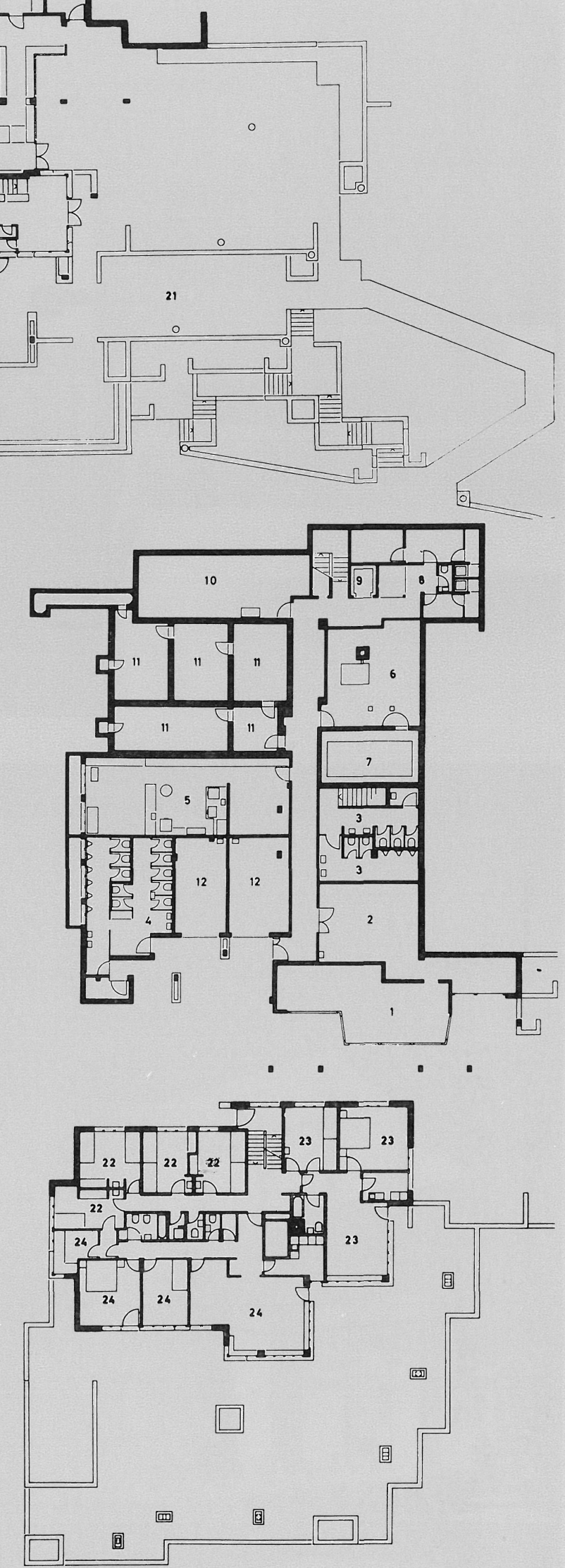

porimerer

22. Cuartos (empleados).-23. Piso del director del kiosco. 24. Piso del director del restaurante. 

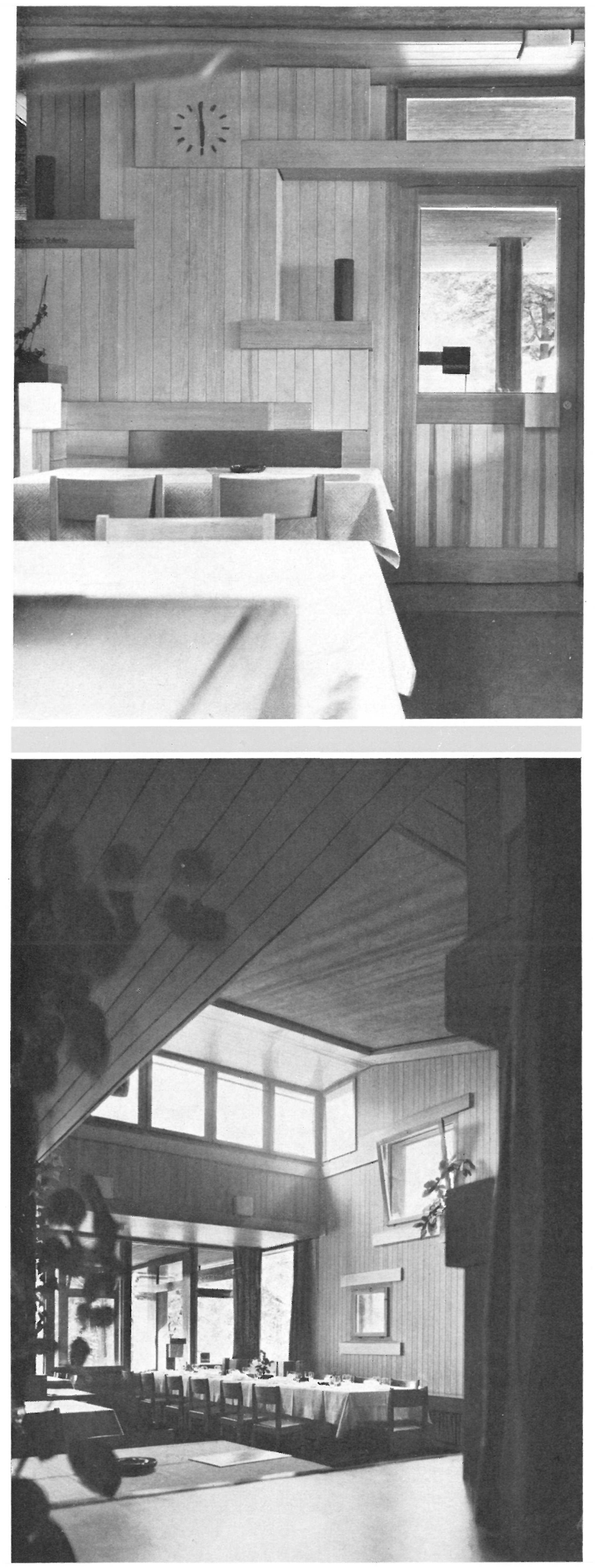

La estructura de la edificación -realizada a base de "hormigón visto», en el que claramente se aprecian las marcas y vetas de las tablas del encofradose eleva gradualmente a partir de la planta situada al nivel de la calle, en la que se distribuyen: un kiosco; los aseos; el guardarropa; bodega para el restaurante; lavadero; el equipo de calefacción; garajes, y un refugio antiaéreo.

En la planta principal están los comedores, cocina, despensas, cámaras y las terrazas-comedor, que dan la sensación de estar prolongadas por la rampa y escalinatas de acceso existentes. Encima de esta planta se encuentra otra que contiene los vestuarios del personal y los despachos de la administración, además de sus correspondientes terrazas; el nivel superior alberga cuartos adicionales para el personal y otro para la maquinaria del ascensor.

Gran parte del atractivo que ofrece este edificio es atribuible al cuidado que pusieron los arquitectos en los más mínimos detalles - accesorios de iluminación, barandillas, jardinería, etcétera-, que forman parte integrante del total de la obra arquitectónica.

La madera ha sido el material empleado predominantemente en los interiores, aunque también aquí se ponen en contraste los dos materiales: madera y hormigón. Así, los cielorrasos han sido dejados con el «hormigón visto», y las paredes del restaurante van revestidas con madera de pino.

Por el contrario, los bancos, las repisas de las ventanas, los tiradores de las puertas, etc., y otros elementos que hubieran sido susceptibles de producir contactos ásperos, han sido realizados a base de tablero contrachapado. El suelo del comedor es de parquet oscuro, y los vestuarios del personal se han revocado y pintado de blanco en sus paramentos verticales y techos, siendo sus suelos revestidos con linóleo. 


\section{Restrumunt "Parle" ì Neulhausen - Suisse}

Foerderer \& Zwimpfer, architectes (Studio: Foerderer \& Otto \& Zwimpfer)

Ce restaurant porte la marque caractéristique de l'architecte-sculpteur Walter Foerderer, avec ses combinaisons béton-bois et ses formes solides et consistantes. L'édifice présente un aspect échelonné bien étudié, résultat de la parfaite adaptation au terrain, ce qui constitue en outre un de ses attraits les plus singuliers.

Ce restaurant est ouvert toute l'année. Dans ses locaux couverts, on peut servir 50 repas, et sur ses terrasses en plein air, utilisées durant la plus grande partie de l'été, on peut accueillir, à chaque service, 200 convives. Il constitue un lieu de halte agréable pour les excursionnistes, qui, de différents points de vue appropriés, peuvent contempler le merveilleux panorama environnant.

"Park" Restaumant, at Meuhausem, Switzeriand

Forderer \& Zwimpfer, architects (Studio: Foerderer \& Otto \& Zwimpfer)

This restaurant exhibits the characteristic style of the architect-sculptor, Walter Foerderer, with its combinations of concrete and timber and solid and strong outlines. The building has a carefully thought stepped up aspect, which follows the ground outline, and this constitutes one of its most singular appeals.

This catering establishment is open all the year round. Its enclosed areas can accommodate 50 clients, and at its outdoor terraces, which are in use most of the year, 200 meals can be served simultaneously. It is a pleasant stopping place for visitors in the course of their excursions, and provides a suitable viewpoint from which to admire the exceptional surrounding panorama.

\section{Park - Restauncumt in Neulhausen - Schmeiz}

Foerderer \& Zwimpfer, Architekten (Studio: Foerderer \& Otto \& Zwimpfer)

Dieser Gaststätte hat der Architekt und Bildhauer Walter Foerderer seinen Stempel -Zusammenspiel von Holz und Beton, massive, stabile Formen- aufgeprägt. Das Gebäude fügt sich vollkommen den Gegebenheiten des Bodens dank seiner gelungenen stufenweisen Gestalt - einer seiner grössten Vorzuige.

Es bleibt das ganze Jahr über geöffnet. Die überdeckten Räume können zur gleichen Zeit 50 Gäste bewirten; auf den Terrassen -in Betrieb fast den ganzen Sommer hindurch- können 200 Gäste pro Turnus bedient werden. Es ist eine angenehme Raststelle für Ausflügler, die ihnen reichlich Aussichten bietet, um die einmalige Landschaft der Umgebung zu geniessen. 